Pacific Journal of Mathematics

ON THE DISTRIBUTIVITY OF THE LATTICE OF FILTERS OF

ORIN FINK AND ROBERT S. SMITH 


\section{ON THE DISTRIBUTIVITY OF THE LATTICE OF FILTERS OF A GROUPOID}

\section{ORRIN Frink AND ROBERT S. SMITH}

In this note we present the results announced in the Notices of the American Mathematical Society, January, 1969. Algebraic lattices are interesting and important algebraic structures. They occur in many branches of algebra, e.g. the lattice of all subalgebras of a universal algebra, the lattice of all filters of a groupoid, and the lattice of all ideals of a ring are all algebraic lattices. Moreover there is a natural connection between algebraic lattices and groupoids, since every algebraic lattice is isomorphic to the lattice of all filters of some groupoid, and in particular of the groupoid of all compact elements of the lattice. If an algebraic lattice is distributive, it is relatively pseudo-complemented and is a complete Brouwerian lattice in the sense of Garrett Birkhoff [1]. Hence it is natural to look for simple conditions on a groupoid that will insure that the lattice of its filters is distributive.

We show that the lattice of all filters is distributive if it is a sublattice of the lattice of all subgroupoids, but this condition is not always necessary for distributivity. If the groupoid is a semilattice, this condition is both necessary and sufficient. We then derive some conditions that are both necessary and sufficient for distributivity for groupoids. One of these is a modification of a condition given by Grätzer and Schmidt for semilattices.

A groupoid is a pair $(G, \tau)$ where $G$ is a set and $\tau$ is a binary operation defined on $G$. We will use the following conventions:

(1) The operation $\tau$ will be called multiplication, and we will write $a b$ for $a \tau b$.

(2) We will write simply $G$ for $(G, \tau)$

(3) The symbol 1 will denote the identity element of $G$ if there is one.

(4) $G^{1}$ will denote the groupoid $G$ with 1 adjoined if $G$ has no identity element; otherwise $G=G^{1}$.

The operation in a groupoid $G$ need not be associative or commutative, hence the elements $a_{1}, \cdots, a_{n}$ have in general many products which can be distinguished from each other by means of parentheses. We find it convenient to denote one of the products of elements $a_{1}$, $\cdots, a_{n}$ by $P\left(a_{1}, \cdots, a_{n}\right)$. We allow the elements to be used more than once and in any order. For example $P_{1}\left(a_{1}, a_{2}\right)$ might be $\left(a_{1} a_{2}\right) a_{1}$, while $P_{2}\left(a_{1}, a_{2}\right)$ could be $a_{2}\left(\left(a_{2} a_{1}\right) a_{1}\right)$. 
Definition. An element $a$ of $G$ is called a factor of element $b$ if there exist elements $a_{1}, \cdots, a_{n}$ of $G$ such that $b=P\left(a_{1}, \cdots, a_{n}, a\right)$.

Definition. A subset $F$ of a groupoid $G$ is called a filter of $G$ if $F$ is a subgroupoid of $G$ which contains all factors of each of its elements.

Proposition. A subset $F$ of a groupoid is a filter if and only if $a b \in F$ is equivalent to $a \in F$ and $b \in F$.

We note that in the theory of algebraic semigroups filters have been studied under the names of co-prime ideals and consistent subsemigroups in the commutative case, and under the names of faces and $p$-semigroups in the general case $[2,5]$.

Let $\mathscr{F}(G)$ denote the collection of all filters of a groupoid $G$, and let $\mathscr{S}(G)$ denote the collection of all subgroupoids of $G$. When only one groupoid $G$ is involved, we will write $\mathscr{F}$ and $\mathscr{S}$ for $\mathscr{F}(G)$ and $\mathscr{S}(G)$ respectively. Observe that $\mathscr{F}$ and $\mathscr{S}$ are algebraic lattices; that is, they are complete lattices in which every element is a join of compact elements. The empty set is the smallest element of both $\mathscr{F}$ and $\mathscr{S}$.

The lattices $\mathscr{F}$ and $\mathscr{S}$ are families of sets, and their elements are subsets of $G$. When $A$ and $B$ are sets, we shall use the notation $A \cup B$ and $A \cap B$ to represent the set union and intersection of $A$ and $B$ respectively. In the lattices $\mathscr{F}$ and $\mathscr{S}$ the lattice meet of two elements $A$ and $B$ is always their set interesection; hence it will be represented by $A \cap B$. The lattice join operations in $\mathscr{F}$ and $\mathscr{S}$ are more complicated.

For the lattice of subgroupoids $\mathscr{S}$, we denote the join of two subgroupoids $A$ and $B$ by $A \vee B$; it is the intersection of all subgroupoids which contain both $A$ and $B$. It consists of all elements of the union $A \cup B$ together with all products involving elements of $A \cup B$.

For the lattice $\mathscr{F}(G)$ of all filters of $G$, we denote the lattice join by $\bar{V}$ and define for $F, H \in \mathscr{F}$ the join $F \bar{V} H$ to be the intersection of all filters containing $F$ and $H$. We later give a more constructive way of obtaining the filter join of two filters, and more generally of the filter generated by any subset of $G$.

An element $a$ of a complete lattice $L$ is said to be compact if $a \leqq \mathrm{~V}\left\{a_{r}: \gamma \in \Gamma\right\}$ implies that there exists a finite subset $F$ of $\Gamma$ such that $a \leqq \mathrm{~V}\left\{a_{r}: \gamma \in F\right\}$. A lattice is said to be compactly generated if every element is a join of compact elements. Lattices which are complete and compactly generated are called algebraic lattices. The lattice of all subalgebras of an algebra with finitary operations is 
an algebraic lattice in which the compact elements are the finitely generated subalgebras. In particular the lattices $\mathscr{S}(G)$ and $\mathscr{F}(G)$ of all subgroupoids and all filters of a groupoid $G$ are algebraic lattices, in which the compact elements are the finitely generated subgroupoids and filters respectively. In the lattice $\mathscr{F}(G)$ every nonempty finitely generated filter is a principal filter generated by a single element, as we shall show.

Definition. A subset $I$ of a groupoid $G$ is an ideal of $G$ if $a \epsilon$ $I$ implies that $a b, b a \in I$ for all $b \in G$. An ideal $I$ of $G$ is said to be prime if $a b \in I$ implies that $a \in I$ or $b \in I$.

The following facts about the lattice $\mathscr{J}(G)$ of all ideals of a groupoid $G$ are easily verified:

(i) $\mathscr{F}(G)$ is a complete ring of sets, closed under arbitrary union and arbitrary intersection.

(ii) A subset $F$ of $G$ is a filter if and only if its complement $G \backslash F$ is a prime ideal.

(iii) The lattice $\mathscr{F}(G)$ of all filters of a groupoid $G$ is distributive if and only if the dual lattice of all prime ideals is distributive.

We find the following notation useful:

$F_{a}$ will denote the filter generated by $a \in G$.

$S_{a}$ will denote the subgroupoid generated by $a \in G$.

$I_{a}$ will denote the ideal generated by $a \in G$.

$F_{A}$ will denote the filter generated by $A \subseteq G$.

$S_{A}$ will denote the subgroupoid generated by $A \subseteq G$.

If $A$ is any subset of a groupoid $G$, then $S_{A}$ consists of the elements of $A$ together with all products of elements of $A$. From this we see that if $S$ and $T$ are subgroupoids of $G$, then their subgroupoid join consists of all elements and all products of the elements of the union $S \cup T$.

We now give a representation of the filter $F_{A}$ generated by a subset $A$ of $G$. This generalizes a result of Petrich for semigroups [5], and will provide us with a representation for the filter join of two filters.

Theorem 1. If $A$ is any subset of a groupoid $G$, then $F_{A}=$ $\bigcup\left\{N_{k}: k \geqq 1\right\}$, where $N_{1}=A$, and $N_{k+1}$ is the subgroupoid generated by $\left\{y \in G: I_{y} \cap N_{k} \neq \varnothing\right\}$ for $k \geqq 1$.

Proof. If $x \in N_{n}$ then $I_{x} \cap N_{n} \neq \varnothing$. Hence $x \in N_{n+1}$ and $N_{n} \subseteq N_{n+1}$. Each $N_{k}$ is a subgroupoid for $k \neq 1$, and the chain union of subgroupoids is a subgroupoid. Therefore the union $\bigcup N_{k}$ is a subgroupoid.

We now show that this union is a filter. If $x y \in \bigcup N_{k}$ then $x y \in N_{n}$ for some $n$. But $x y \in I_{x}$ and $x y \in I_{y}$, whence $x y \in I_{x y} \subseteq I_{x} \cap I_{y}$. 
Theorefore $\varnothing \neq N_{n} \cap I_{x} \cap I_{y}$, and hence $N_{n} \cap I_{y} \neq \varnothing \neq N_{n} \cap I_{x}$, and so $x, y \in N_{n+1} \subseteq \bigcup N_{k}$. Hence this union is a filter.

To complete the proof we show that $\bigcup N_{k} \subseteq F_{A}$. We do this by induction. Clearly $A=N_{1} \subseteq F_{A}$. Assume that $N_{n} \subseteq F_{A}$ and show that $N_{n+1} \subseteq F_{A}$. If $z \in I_{y} \cap N_{n}$, then $y$ is a factor of $z$ and since $z \in$ $N_{n} \subseteq F_{A}$, all factors of $z$ are in $F_{A}$. Hence $y \in F_{A}$ and thus $\{w \in$ $\left.G: I_{w} \cap N_{n} \neq \varnothing\right\} \subseteq F_{A}$. Hence $N_{n+1}$, which is the subgroupoid generated by this set, is a subset of $F_{A}$, completing the induction. Therefore $\mathrm{U} N_{k} \subseteq F_{A}$. This completes the proof.

Before giving a characterization of the filter join $F \bar{V} H$ of two filters, we call attention to some facts about the lattice of filters $\mathscr{F}$ which are easily verified. First we note that $F_{x y}=F_{x} \bar{V} F_{y}$. It follows that the join of any finite number of principal filters is a principal filter. Hence every finitely generated filter is principal, with the exception of the empty filter.

Let $\mathscr{C}(G)=\left\{F_{x}: x \in G\right\}$ denote the set of all principal filters of $G$. Then $\mathscr{C}(G)$ is an upper semilattice with respect to the operation $\bar{V}$ of filter join. The mapping $x \rightarrow F_{x}$ is a homomorphism of $G$ onto $\mathscr{C l}(G)$, and $\mathscr{C}(G)$ is the maximal semilattice homomorphic image of $G$. The existence of such a maximal semilattice homomorph of a groupoid is well known; see [2] page 18, [5] page 70. We state these facts as a theorem.

Theorem 2. Every groupoid $G$ has a maximal semilattice homomorphic image $\mathscr{C}(G)$, consisting of the principal filters of $G$.

The semilattice $\mathscr{C}(G)$ may not have an identity element, since the empty filter is not a principal filter. If $G$ has an identity element 1 , then $F_{1}$ is the identity element of $\mathscr{C}(G)$. In an upper semilattice an identity element is usually denoted by 0 , and a filter is called an ideal. Our definition of ideal is that used in the theory of semigroups and groupoids. Note that $\mathscr{C}(G)$ is not in general a lattice, since the intersection of two principal filters need not be principal.

We now define two important binary relations in a groupoid.

Definition. In a groupoid $G$ we define $a \subset b$ to mean $F_{a} \subseteq F_{b}$, and $a \sim b$ to mean $F_{a}=F_{b}$.

Note that the relation $a \subset b$ is reflexive and transitive and hence a quasi-ordering. In general it is not a relation of partial order, since from $a \subset b$ and $b \subset a$ follows only $a \sim b$ but not always $a=b$. We note that $a \subset b$ is equivalent to $a \in F_{b}$. We now give a characterization of the join of two filters.

THEOREM 3. If $F$ and $H$ are two filters of $G$, then $F \bar{\nabla} H=$ $\left\{a \in G: a \subset P\left(b_{1}, \cdots, b_{n}\right)\right.$, where $\left.b_{1}, \cdots, b_{n} \in F \cup H\right\}$. 
Proof. Let $a \in F \bar{V} H$. Then $F_{a} \leqq \bar{\nabla}\left\{F_{b}: b \in F \cup H\right\}$. But $F_{a}$ is a compact element of the lattice $\mathscr{F}(G)$. Hence there exist elements $b_{1}, \cdots, b_{n}$ in $F \cup H$ such that $F_{a} \subseteq F_{b_{1}} \vee \cdots \vee F_{b_{n}}$. Hence $a \in F_{\circ}$ where $c=P\left(b_{1}, \cdots, b_{n}\right)$, whence $a \subset c$.

Conversely, let $a \subset P\left(b_{1}, \cdots, b_{n}\right)$, with $b_{1}, \cdots, b_{n} \in F \cup H$. Then $a \in F_{c}$, where $c=P\left(b_{1}, \cdots, b_{n}\right), F_{c} \leqq F \bar{\nabla} H$, hence $a \in F \bar{\nabla} H$. This, completes the proof.

We note that $\sim$ is a congruence relation on $G$, and that the quotient $G / \sim$ is the semilattice $\mathscr{C}(G)$. If $a \subset b$, it does not follow that $a$ is a factor of $b$; but the converse is true. However, a subgroupoid $T$ is a filter of $G$ if and only if $x \subset y$ and $y \in T$ implies that $x \in T$.

Now that we have characterized the lattice operations in the lattice of filters $\mathscr{F}(G)$, we look for conditions for the distributivity of this lattice. If $G$ is a semilattice, it will be shown that $\mathscr{F}(G)$ is distributive if and only if it is a sublattice of $\mathscr{S}(G)$. For groupoids in general, this condition is sufficient but not necessary.

Definition. An element $a$ of a lattice $(L, \wedge, \vee)$ is called meetdistributive if $a \wedge(b \vee c)=(a \wedge b) \vee(a \wedge c)$ for all $b, c$ in $L$.

THEOREM 4. Every filter of a groupoid $G$ is a meet-distributive element of the lattice $\mathscr{S}(G)$ of subgroupoids of $G$.

Proof. Let $F$ be a filter and $S$ and $T$ subgroupoids. It is sufficient to show that $F \cap(S \vee T) \cong(F \cap S) \vee(F \cap T)$. Suppose $x \in F \cap(S \vee T)$. Since $x \in S \vee T, x=P\left(a_{1}, \cdots, a_{n}\right)$ where $a_{1}, \cdots, a_{n}$ are in $S \cup T$. Since $x \in F$ and $F$ is a filter, each of the elements $a_{1}, \cdots$, $a_{n}$ is in $F$. It follows that $x \in(F \cap S) \vee(F \cap T)$. This ends the proof.

COROLlary. If the lattice of filters $\mathscr{F}$ is a sublattice of the lattice of subgroupoids $\mathscr{S}$, then $\mathscr{F}$ is distributive.

However, the condition that $\mathscr{F}$ is a sublattice of $\mathscr{S}$ is not necessary for the distributivity of $\mathscr{F}$, as is shown by the following example of a groupoid $G=\{x, y, z, u\}$, with multiplication table:

\begin{tabular}{l|llll}
\multicolumn{1}{c}{} & $x$ & $y$ & $z$ & $u$ \\
\cline { 2 - 4 }$x$ & $x$ & $z$ & $z$ & $u$ \\
$y$ & $z$ & $y$ & $z$ & $u$ \\
$z$ & $z$ & $z$ & $z$ & $u$ \\
$u$ & $u$ & $u$ & $u$ & $z$
\end{tabular}.


Here the lattice of filters $\mathscr{F}(G)$ is a well-known distributive lattice with the four elements $\varnothing, F_{x}, F_{y}$, and $F_{z}=F_{u}$. The subgroupoid join $F_{x} \vee F_{y}$ is the set $\{x, y, z\}$, while the filter join $F_{x} \bar{\vee} F_{y}$ is the set $\{x, y, z, u\}$. Hence the lattice $\mathscr{F}(G)$ is not a sublattice of $\mathscr{S}(G)$. Note that $G$ is a commutative semigroup, but not a semilattice, since $u^{2} \neq$ $u$. For semilattices it will be shown that the sublattice condition is also necessary for distributivity.

We now give another characterization of the filter join of two filters.

Theorem 5. If $A$ and $B$ are filters of a groupoid $G$, then (1) $A \bar{\vee} B=A \cup B \cup\{x: x \subset a b, a \in A, b \in B\}$.

Proof. The right side of (1) is a subset of the left side, since $A \bar{\vee} B$ is a filter containing $A \cup B$. To show the reverse inclusion, suppose $x \in A \bar{\vee} B$. By Theorem 3, we have $x \subset P\left(c_{1}, \cdots, c_{n}\right)$, where $\left\{c_{1}, \cdots, c_{n}\right\} \subseteq A \cup B$. Hence $x \in F_{P}=\bar{V}\left\{F_{c}: c \in\left\{c_{1}, \cdots, c_{n}\right\}\right\}$. If all the elements $c_{i}$ are in $A$ or in $B$, then so is $x$, and $x$ is a member of the right side of (1). If not, then $F_{P}=F_{a} \bar{V} F_{b}$, where $a$ is a product of the elements $c_{i}$ in $A$, and $b$ is a product of the elements $c_{i}$ in $B$. But $F_{a} \bar{\vee} F_{b}=F_{a b}$, hence $x \in F_{a b}$ and $x \subset a b$, where $a \in A, b \in B$. Hence $x$ is in the right side of (1), which completes the proof.

We now give a necessary and sufficient condition for the distributivity of $\mathscr{F}(G)$ in terms of a relation between the filter join and the subgroupoid join of two filters.

Theorem 6. The filter lattice $\mathscr{F}(G)$ of a groupoid $G$ is distributive if and only if to each element $x$ of the filter join $A \bar{\vee} B$ of two filters there exists an element $y$ of the subgroupoid join $A \vee B$ such that $x \sim y$.

Proof. To prove sufficiency, let $x \in A \cap(B \bar{\vee} C)$, where $A, B, C$ are filters of $G$. We must show that $x \in(A \cap B) \bar{V}(A \cap C)$. Now $x \in A$, and by the condition of the theorem there exists $y$ in $B \vee C$ such that $x \sim y$. Note that $x \sim y$ holds if and only if $x$ and $y$ are in the same filters. Hence $y \in A$, and $y \in A \cap(B \vee C)$. By Theorem 4, $A$ is a meet-distributive element of $\mathscr{S}(G)$. Hence $A \cap(B \vee C)=$ $(A \cap B) \vee(A \cap C) \subseteq(A \cap B) \bar{\vee}(A \cap C)$. It follows that $y$, and hence $x$, is in the filter $(A \cap B) \bar{V}(A \cap C)$, which proves the sufficiency of the condition.

To prove the necessity, suppose $\mathscr{F}(G)$ is distributive and $x \in$ $A \bar{\vee} B$, where $A$ and $B$ are filters. We must show that $x \sim y$, where $y \in A \vee B$. Since $x \in F_{x} \cap(A \bar{\vee} B)$, by distributivity $x \in\left(F_{x} \cap A\right) \bar{\vee}$ 
$\left(F_{x} \cap B\right)$. By Theorem 5, either $x \in A \cup B$, whence the conclusion would follow with $x=y$, or $x \subset a b$ with $a \in F_{x} \cap A$ and $b \in F_{x} \cap B$. Then $a \in A, b \in B$, hence $a b \in A \vee B$. Also $a \in F_{x}, b \in F_{x}$ hence $a b \in F_{x}$ and $a b \subset x$. But also $x \subset a b$. Hence $x \sim a b$. Let $y=a b$. Then $y \in A \vee$ $B$ and $x \sim y$, which was to be proved.

We now show that the sublattice condition is both necessary and sufficient for distributivity of $\mathscr{F}(G)$ if $G$ is a semilattice.

TheoRem 7. The filter lattice $\mathscr{F}(G)$ of a semilattice $G$ is distributive if and only if it is a sublattice of the lattice $\mathscr{S}(G)$ of all subsemilattices of $G$.

Proof. The sufficiency of the condition follows from the Corollary to Theorem 4. To prove the necessity, suppose $\mathscr{F}(G)$ is distributive and $A$ and $B$ are filters of $G$. We must show that $A \bar{\vee} B \cong$ $A \vee B$. Suppose $x \in A \bar{\vee} B$. By Theorem 6 there exists an element $y$ in $A \vee B$ such that $x \sim y$. It is well known that in a semilattice the relation $x \subset y$ is one of partial order rather than merely a quasiorder. In fact, $x \subset y$ if and only if $x y=y$, and $y \subset x$ if and only if $x y=x$. If $x \sim y$, then $x \subset y$ and $y \subset x$, hence $x=y$. It follows that $x \in A \vee B$, and hence $A \bar{\vee} B=A \vee B$. Then $\mathscr{F}(G)$ is a sublattice of $\mathscr{S}(G)$, which was to be proved.

We now derive a necessary and sufficient condition for the distributivity of $\mathscr{F}(G)$ which is somewhat similar to a condition of Grätzer and Schmidt for upper semilattices $(S, \vee)$. The original GrätzerSchmidt condition is:

If $a \leqq b \vee c$, then there exist in $S$ elements $b^{\prime}, c^{\prime}$ such that $b^{\prime} \leqq$ $b, c^{\prime} \leqq c$, and $a=b^{\prime} \vee c^{\prime}$.

This condition is referred to in the paper [6] by E. T. Schmidt, and is studied in the unpublished paper [3] by Grätzer and Schmidt. It is sufficient for the distributivity of $\mathscr{F}(S)$, which is called the lattice of ideals if $S$ is a semilattice. If $S$ has in identity element 0 , the Grätzer-Schmidt condition is also necessary for distributivity. Unfortunately it is not always necessary if $S$ has no zero element, as will be shown by an example.

For a groupoid $G$, the Grätzer-Schmidt condition takes the following form:

If a $\subset b c$, then there exist in $G$ elements $b^{\prime}, c^{\prime}$ such that $b^{\prime} \subset b$, $c^{\prime} \subset c$, and $a \sim b^{\prime} c^{\prime}$.

Again this condition is sufficient for the distributivity of $\mathscr{F}(G)$, and is also necessary if $G$ has an identity element. The following condition is more complicated, but is always both necessary and sufficient for distibutivity of $\mathscr{F}(G)$ : 
If $a \subset b c$, then either $a \subset b$, or $a \subset c$, or there exist elements $b^{\prime}$, $c^{\prime}$ of $G$ such that $b^{\prime} \subset b, c^{\prime} \subset c$, and $a \sim b^{\prime} c^{\prime}$.

THEOREM 8. The lattice of filters $\mathscr{F}(G)$ is distributive if and only if whenever $a \subset b c$, then either $a \subset b$, or $a \subset c$, or there exist elements $b^{\prime}, c^{\prime}$ of $G$ such that $b^{\prime} \subset b, c^{\prime} \subset c$, and $a \sim b^{\prime} c^{\prime}$.

Proof. To prove sufficiency, suppose the condition holds, and $x \in A \bar{\vee} B$, where $A$ and $B$ are filters of $G$. By Theorem 6 we must show that $x \sim y$ for some element $y$ in $A \vee B$. By Theorem 5, either $x$ is in $A \cup B$, in which case $y=x$ is in $A \vee B$, or $x \subset a b$ with $a \in A$ and $b \in B$. In the latter case by the hypothesis of our theorem either $x \subset a$ or $x \subset b$, in which case $y=x$ is in $A \vee B$, or there exist elements $a^{\prime} \subset a$ and $b^{\prime} \subset b$ such that $x \sim a^{\prime} b^{\prime}$. Since $A$ and $B$ are filters, $a^{\prime} \in A$ and $b^{\prime} \in B$. Hence $a^{\prime} b^{\prime} \in A \vee B$. Let $y=a^{\prime} b^{\prime}$. Then $x \sim y$ and $y \in A \vee B$. This proves the sufficiency of the condition.

To prove necessity, suppose the lattice $\mathscr{F}(G)$ is distributive, and $a \subset b c$. Then $F_{a} \subseteq F_{b c}=F_{b} \bar{\vee} F_{c}$. It follows that $a \in F_{a} \cap$ $\left(F_{b} \bar{\vee} F_{c}\right)$. By distributivity, we see that $a \in\left(F_{a} \cap F_{b}\right) \bar{V}\left(F_{a} \cap F_{c}\right)$. Then by Theorem 5 either $a \in F_{b}$, whence $a \subset b$, or $a \in F_{c}$, whence $a \subset c$, or $a \subset b^{\prime} c^{\prime}$, where $b^{\prime} \in F_{a} \cap F_{b}$, and $c^{\prime} \in F_{a} \cap F_{c}$. In the latter case it follows that $b^{\prime} \subset b, c^{\prime} \subset c$, and $b^{\prime} c^{\prime} \subset a$. Since also we have $a \subset b^{\prime} c^{\prime}$, it follows that $a \sim b^{\prime} c^{\prime}$. But this is just the condition of the theorem, and the proof ends.

The Grätzer-Schmidt condition is not always necessary for the distributivity of $\mathscr{F}$ even for semilattices, as the following example shows. Consider the three-element semilattice $G=\{x, y, z\}$ in which $x y=x z=y z=z$; note that $G$ has no identity element. The lattice $\mathscr{F}(G)$ consists of the four elements $\varnothing, F_{x}, F_{y}, F_{z}$ and is distributive. Now $x \subset x y$, but there do not exist elements $x^{\prime} \subset x$ and $y^{\prime} \subset y$ such that $x \sim x^{\prime} y^{\prime}$. For if $x^{\prime} \subset x$, then $x^{\prime}=x$, and if $y^{\prime} \subset y$, then $y^{\prime}=y$. Hence $x^{\prime} y^{\prime}=z$, but $x \sim z$ is false, and condition fails.

However, for groupoids with an identity element, and more generally for groupoids which are lcwer-directed, the Grätzer-Schmidt condition is both necessary and sufficient for the distibutivity of $\mathscr{F}$.

Definition. A groupoid $G$ is said to be lower-directed if given any two elements $a$ and $b$ of $G$, there exists an element $x$ of $G$ such that $x \subset a$ and $x \subset b$.

Note that $G$ is lower-directed if and only if the filter lattice $\mathscr{F}(G)$ has the finite intersection property; this means that the intersection of any two, and hence of any finite number of nonempty filters is a nonempty filter. Note also that a groupoid with an identity 
element 1 is lower-directed, since the element 1 is a factor of every element, hence $1 \subset x$ for all $x$ in $G$.

Theorem 9. The filter lattice $\mathscr{F}(G)$ of a lower-directed groupoid $G$, and hence of a groupoid with identity element, is distributive if and only if the Grätzer-Schmidt condition holds, that is, if $a \subset b c$, then there exist elements $b^{\prime} \subset b$ and $c^{\prime} \subset c$ in $G$ such that $a \sim b^{\prime} c^{\prime}$.

Proof. The condition is sufficient, since the Grätzer-Schmidt condition is stronger than and implies the condition of Theorem 8 . To show that it is also necessary in this case, suppose $\mathscr{F}(G)$ is distributive and $a \subset b c$. Then by Theorem 8 either elements $b^{\prime}$ and $c^{\prime}$ with the required properties exist, or $a \subset b$ or $a \subset c$. The argument is the same either way, so suppose $a \subset b$. Since $G$ is lower-directed, there exists an element $x$ in $G$ such that $x \subset a$ and $x \subset c$. Then $a x \subset a a$. Let $b^{\prime}=a$ and $c^{\prime}=x$. Then $b^{\prime} \subset b, c^{\prime} \subset c$, and $b^{\prime} c^{\prime}=a x$. But $a \subset a x \subset a a \subset a$, hence $a \sim a x$ and $a \sim b^{\prime} c^{\prime}$. Hence the conclusion of the Grätzer-Schmidt condition holds, and the condition is also necessary, ending the proof.

Note that a groupoid with the Grätzer-Schmidt property is necessarily lower-directed. For if $a$ and $b$ are any two elements of $G$, then $a \subset a b$, hence elements $a^{\prime} \subset a$ and $b^{\prime} \subset b$ exist with $a \sim a^{\prime} b^{\prime}$. Then $b^{\prime}$ is a common lower bound of $a$ and $b$, since $b^{\prime} \subset b$, and $b^{\prime} \subset$ $a b \subset a$. Hence $G$ is lower-directed.

We now apply Theorem 9 to obtain a necessary and sufficient condition for the distributivity of an arbitrary algebraic lattice. This result is not really new, since it was known some time ago to Grätzer and Schmidt.

THEOREM 10. An algebraic lattice $(L, \wedge, \vee)$ is distributive if and only if whenever $a, b, c$ are compact elements of $L$, and $a \leqq b \vee c$, then there exist compact elements $b^{\prime}$ and $c^{\prime}$ such that $b^{\prime} \leqq b, c^{\prime} \leqq c$, and $a=b^{\prime} \vee c^{\prime}$.

Proof. Since $L$ is complete, it has a least element 0 which is compact. The compact elements of $L$ form an upper semilattice $(G$, $\checkmark)$ with identity element 0 . It is well known that $L$ is isomorphic to $\mathscr{F}(G)$, which is usually called the lattice of ideals of $G$. By Theorem $9, L$ is distributive if and only if the Grätzer-Schmidt condition holds in $G$. The condition of the theorem is the form this condition takes in an upper semilattice. This ends the proof.

We now give another necessary and sufficient condition for the distributivity of the lattice $\mathscr{F}(G)$. 
THEOREM 11. The lattice $\mathscr{F}(G)$ of a groupoid $G$ is distributive if and only if the Grätzer-Schmidt condition holds in $G^{1}$.

Proof. If $G$ has an identity element 1 , then $G=G^{1}$ and the result follows from Theorem 9 . If not, then $G^{1}$ is formed by adjoining to $G$ a single element 1, which is a factor of every element of $G^{1}$ and a member of every nonempty filter of $G^{1}$. Hence $\mathscr{F}(G)$ is isomorphic to the lattice of all nonempty filters of $G^{1}$, and is distributive if and only if $\mathscr{F}\left(G^{1}\right)$ is distributive. By Theorem 9, this is true if and only if the Grätzer-Schmidt condition holds in $G^{1}$.

Since every algebraic lattice, and hence every finite lattice, is isomorphic to some filter lattice $\mathscr{F}(G)$, filter lattices in general are neither modular or distributive.

\section{REFERENCES}

1. G. Birkhoff, Lattice Theory, 3d ed., The Amer. Math. Soc. Providence, 1967.

2. A. H. Clifford and G. B. Preston, The Algebraic Theory of Semigroups, vol. 1, The Amer. Math. Soc., Providence, 1961.

3. G. Grätzer and E. T. Schmidt, On distributive semilattices, unpublished.

4. L. Nachbin, A characterization of the lattice of all ideals of a Boolean ring, Funda. Math., 36 (1949), 137-142.

5. M. Petrich, The maximal semilattice decomposition of a semigroup, Math. Z., 85 (1964), 68-82.

6. E. T. Schmidt, Über die Kongruenzverbände der Verbände, Publ. Math. Debrecen 9 (1962), 243-256.

Received June 11, 1971. 


\section{PACIFIC JOURNAL OF MATHEMATICS}

\section{EDITORS}

\author{
H. SAMELSON \\ Stanford University \\ Stanford, California 94305 \\ C. R. HOBBY \\ University of Washington \\ Seattle, Washington 98105
}

\section{J. DuGundJI}

Department of Mathematics University of Southern California Los Angeles, California 90007

RICHARD ARENS

University of California

Los Angeles, California 90024

\section{ASSOCIATE EDITORS}
E. F. BECKENBACH
B. H. NeumanN
F. WOLF
K. YOSHIDA

\section{SUPPORTING INSTITUTIONS}

\author{
UNIVERSITY OF BRITISH COLUMBIA \\ CALIFORNIA INSTITUTE OF TECHNOLOGY \\ UNIVERSITY OF CALIFORNIA \\ MONTANA STATE UNIVERSITY \\ UNIVERSITY OF NEVADA \\ NEW MEXICO STATE UNIVERSITY \\ OREGON STATE UNIVERSITY \\ UNIVERSITY OF OREGON \\ OSAKA UNIVERSITY
}

\author{
UNIVERSITY OF SOUTHERN CALIFORNIA \\ STANFORD UNIVERSITY \\ UNIVERSITY OF TOKYO \\ UNIVERSITY OF UTAH \\ WASHINGTON STATE UNIVERSITY \\ UNIVERSITY OF WASHINGTON \\ AMERICAN MATHEMATICAL SOCIETY \\ NAVAL WEAPONS CENTER
}

The Supporting Institutions listed above contribute to the cost of publication of this Journal, but they are not owners or publishers and have no responsibility for its content or policies.

Mathematical papers intended for publication in the Pacific Journal of Mathematics should be in typed form or offset-reproduced, (not dittoed), double spaced with large margins. Underline Greek letters in red, German in green, and script in blue. The first paragraph or two must be capable of being used separately as a synopsis of the entire paper. The editorial "we" must not be used in the synopsis, and items of the bibliography should not be cited there unless absolutely necessary, in which case they must be identified by author and Journal, rather than by item number. Manuscripts, in duplicate if possible, may be sent to any one of the four editors. Please classify according to the scheme of Math. Rev. Index to Vol. 39. All other communications to the editors should be addressed to the managing editor, Richard Arens, University of California, Los Angeles, California, 90024.

50 reprints are provided free for each article; additional copies may be obtained at cost in multiples of 50 .

The Pacific Journal of Mathematics is published monthly. Effective with Volume 16 the price per volume (3 numbers) is $\$ 8.00$; single issues, $\$ 3.00$. Special price for current issues to individual faculty members of supporting institutions and to individual members of the American Mathematical Society: $\$ 4.00$ per volume; single issues $\$ 1.50$. Back numbers are available.

Subscriptions, orders for back numbers, and changes of address should be sent to Pacific Journal of Mathematics, 103 Highland Boulevard, Berkeley, California, 94708.

PUBLISHED BY PACIFIC JOURNAL OF MATHEMATICS, A NON-PROFIT CORPORATION

Printed at Kokusai Bunken Insatsusha (International Academic Printing Co., Ltd.), 270, 3-chome Totsuka-cho, Shinjuku-ku, Tokyo 160, Japan. 


\section{Pacific Journal of Mathematics}

\section{Vol. 42, No. $2 \quad$ February, 1972}

Stephen Richard Bernfeld, The extendability of solutions of perturbed scalar differential equations ................................. 277

James Edwin Brink, Inequalities involving $f_{-} p$ and $f^{(n)}{ }_{q}$ for $f$ with $n$

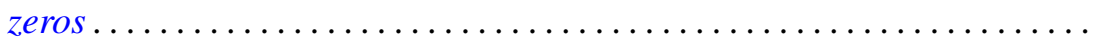

Orrin Frink and Robert S. Smith, On the distributivity of the lattice of filters of a groupoid

Donald Goldsmith, On the density of certain cohesive basic sequences .... 323

Charles Lemuel Hagopian, Planar images of decomposable continua . . . . . 329

W. N. Hudson, A decomposition theorem for biadditive processes ........ 333

W. N. Hudson, Continuity of sample functions of biadditive processes......

Masako Izumi and Shin-ichi Izumi, Integrability of trigonometric series.

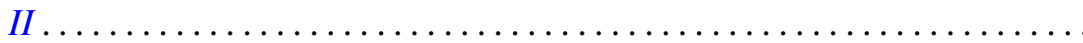

H. M. Ko, Fixed point theorems for point-to-set mappings and the set of

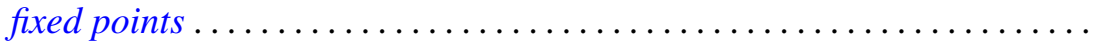

Gregers Louis Krabbe, An algebra of generalized functions on an open interval: two-sided operational calculus ...

Thomas Latimer Kriete, III, Complete non-selfadjointness of almost selfadjoint operators.................................

Shiva Narain Lal and Siya Ram, On the absolute Hausdorff summability of a Fourier series .

Ronald Leslie Lipsman, Representation theory of almost connected groups...

James R. McLaughlin, Integrated orthonormal series.... . .

H. Minc, On permanents of circulants.

Akihiro Okuyama, On a generalization of $\Sigma$-spaces.....

Norberto Salinas, Invariant subspaces and operators of class $(S)$

James D. Stafney, The spectrum of certain lower triangular matrices as operators on the $l_{p}$ spaces .......................

Arne Stray, Interpolation by analytic functions

$\mathrm{Li} \mathrm{Pi} \mathrm{Su}$, Rings of analytic functions on any subset of the complex plane.

R. J. Tondra, A property of manifolds compactly equivalent to compact manifolds.... 